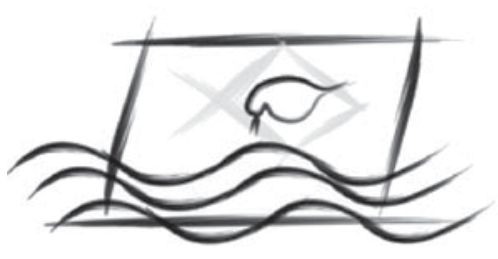

ECOTOX - Brazil
J. Braz. Soc. Ecotoxicol., v. 4, n. 1-3, 2009, 15-20

doi: $10.5132 /$ jbse.2009.01.003

\title{
Revisão da Metodologia e Sensiblidade do Tanaidáceo Kalliapseudes schubartii em Ensaios com Substâncias de Referência
}

\author{
L. S. M. Mottola*, G. Schork \& C. Resgalla JR. \\ CTTMar/UNIVALI, CP 360, CEP 88.302-202, Itajaí, SC
}

(Received September 19, 2007; Accepted March 3, 2008)

\begin{abstract}
RESUMO
No Brasil, avaliações da qualidade de sedimentos vêm ganhando maior atenção após a resolução do CONAMA n 344 de 2004, que regulariza os níveis de contaminantes em materiais dragados, levando a uma maior investigação da sensibilidade e na definição dos protocolos dos testes aplicados aos organismos bentônicos. Em função disto, este trabalho teve como objetivo revisar a metodologia do teste de toxicidade aguda com o tanaidáceo Kalliapseudes schubartii assim como investigar sua sensibilidade frente a tóxicos de referência normalmente utilizados na ecotoxicologia marinha. Foram realizados experimentos de sensibilidade com as substâncias $\mathrm{Zn}, \mathrm{Cd}$, DSS (dodecil sulfato de sódio) e $\mathrm{NH}_{3}$ em duas condições de salinidade (grupo 1: salinidade 15 e grupo 2: salinidades ambientais de 5,$7 ; 7,3 ; 8,5 ; 10$ e 21). Nestes experimentos, mortalidade e mobilidade foram observadas como efeitos. A partir da obtenção da $\mathrm{CE}_{50}$ e $\mathrm{CL}_{50}$ incipientes, observou-se que são necessários 6 dias para a realização dos testes de mobilidade e 7 dias para os de mortalidade. Para o zinco, nos testes com os indivíduos aclimatados em salinidade 15 (grupo 1) foram encontrados resultados com maior replicabilidade. Para o grupo 1, obteve-se $\mathrm{CL}_{50}(7 \mathrm{dias})$ : $\mathrm{Zn}=7,75 \mathrm{mg} \mathrm{L}^{-1} ; \mathrm{Cd}=0,29 \mathrm{mg} \mathrm{L}^{-1}$; DSS $=12,12 \mathrm{mg} \mathrm{L}^{-1}$ e amônia não ionizada $=3,16 \mathrm{mg} \mathrm{L}^{-1}$. Os resultados do grupo 2 demonstraram que o processo de aclimatação dos organismos pode interferir na sua sensibilidade, entretanto, sem um padrão aparente bem definido. $\mathrm{O}$ end point mobilidade mostrou-se menos preciso e por isso não apresentou vantagens em relação ao uso da mortalidade. De forma geral, o tanaidáceo mostrou-se menos sensível que outros organismos teste utilizados na avaliação da qualidade do sedimento, por outro lado, apresenta algumas importantes vantagens como a sua ampla tolerância à salinidade, preferência por sedimentos finos e alta resistência à amônia não ionizada.
\end{abstract}

Palavras-chave: Kalliapseudes schubartii, sedimento, tanaidacea, tóxico de referência.

\begin{abstract}
Review of the protocol and sensibility of the tanaid Kalliapseudes schubartii in tests conducted with reference toxicants

Since CONAMA resolution 344 (2004), which regulates the levels of contaminants in dredged material, the evaluation of the quality of sediments has received increased attention in Brazil in an attempt to establish standards for test protocols applied to benthic organisms and to investigate the sensitivity of this species. Regarding this issue, the present investigation had to main objectives. Firstly, to review test protocols for acute toxicity using the tanaidacea Kalliapseudes schubartii, and secondly to investigate the sensitivity of this species for reference toxicants usually employed in the marine ecotoxicology. Sensitivity experiments were carried out with the substances $\mathrm{Zn}, \mathrm{Cd}$, DSS (sodium dodecyl sulfate) and $\mathrm{NH}_{3}$ in two salinity conditions (group 1: salinity 15 and group 2: local salinity $5.7 ; 7.3 ; 8.5 ; 10$ e 21). In these experiments, mortality and mobility were assessed. Based on the threshold $\mathrm{EC}_{50}$ and $\mathrm{LC}_{50}$, six days were needed to observe significant mobility effects, while seven days were needed to observed significant mortality effects. For zinc, in tests with individuals acclimatized in salinity 15 (group 1) there were found results with higher reproducibility. For group 1, were obtained $\mathrm{LC}_{50}$ (7 days): $\mathrm{Zn}=7.75 \mathrm{mg} \mathrm{L}^{-1} ; \mathrm{Cd}=0.29 \mathrm{mg} \mathrm{L}^{-1}$; DSS $=12.12 \mathrm{mg} \mathrm{L}^{-1}$ and nonionized ammonia $=3.16 \mathrm{mg} \mathrm{L}^{-1}$. The results obtained to group 2 demonstrated that the acclimatization process of the organisms must interfere in their sensitivity, but, without a well defined visible pattern. The mobility as end point was less precise and therefore did not present advantages on the use of the mortality. In tests intended to evaluate the quality of the sediment, tanaids were generally found to be less sensitive than other organisms. However, using tanaids presented some advantages such as their wide salinity tolerance, preference for fine sediments, and high resistance to non-ionized ammonia.
\end{abstract}

Keywords: Kalliapseudes schubartii, sediment, tanaidacea, reference toxicant.

* Corresponding author: Letícia Salua Maraschin Mottola, e-mail: salua03@yahoo.com.br. 


\section{INTRODUÇÃO}

No Brasil, os testes de toxicidade com sedimento ganharam mais atenção devido à recente resolução do CONAMA n 344 de 2004 que estabelece as diretrizes gerais e os procedimentos mínimos para a avaliação do material a ser dragado. Entretanto, diferentes metodologias podem ser empregadas em testes que avaliam a toxicidade dos sedimentos. Experimentos com a fase sólida do sedimento apresentam uma duração de 10 dias em testes letais e utilizam principalmente macroinvertebrados bentônicos, como anfípodos e poliquetos, tanto adultos como juvenis. No Brasil, os organismos teste normalmente utilizados são os anfípodas Tiburonella viscana (Abessa et al., 1998; Abessa et al., 2001) e Hyalella azteca (Portela et al., 2006). Entretanto, a necessidade de encontrar outros organismos com características mais adequadas para avaliar sedimentos estuarinos, como ampla tolerância às variações de salinidade, levou ao desenvolvimento de pesquisas com o tanaidáceo Kalliapseudes schubartii (Zamboni \& Costa, 2002).

Típico de zonas mixohalinas, o tanaidáceo tubícola $K$. schubartii é uma das espécies mais abundantes em ambientes estuarinos da costa sul e sudeste brasileira (Rosa \& Bemvenuti, 2006; Pagliosa \& Barbosa, 2006; Leite, 1995). Possui hábito infaunal suspensívoro-detritivoro, apresenta distribuição agregada e concentra-se na camada superficial do substrato, estando associado com sedimentos finos e moderadamente selecionados e com teores elevados de matéria orgânica (Leite et al., 2003). Apresenta alta importância ecológica, desempenhando um papel importante na cadeia trófica do estuário como item alimentar de espécies com reconhecido valor econômico (Figueiredo \& Vieira, 2005; Fonseca \& D'Incao, 2006).

Sua utilização como organismo teste na avaliação da qualidade dos sedimentos já vem sendo testada, assim como a sua sensibilidade frente a uma série de substâncias tóxicas (Costa, 1997). Contudo, para a realização destes experimentos, tem sido utilizada como referência protocolar a metodologia padronizada pela ASTM (1995) para o anfípodo Hyalella azteca, incluindo modificações somente no volume de sedimento e na salinidade do experimento (Zamboni \& Costa, 2002). Devido a pouca informação sobre este tipo de experimento, o objetivo deste trabalho foi revisar a metodologia que vem sendo empregada nos testes de toxicidade aguda com o uso do tanaidáceo $K$. schubartii em experimentos com substâncias de referência, além de comparar a sua sensibilidade em diferentes condições salinas, bem como com outros organismos rotineiramente utilizados em testes com sedimento total.

\section{MATERIAL E MÉTODOS}

\section{Coleta dos organismos e aclimatação}

Os organismos foram coletados nas proximidades da desembocadura do rio Itajaí-Açu (litoral norte de Santa Catarina), na margem do município de Navegantes, em uma profundidade aproximada de 0,5 metros. Com auxílio de uma pá, retirou-se uma camada de sedimento de aproximadamente
$15 \mathrm{~cm}$ de espessura que foi peneirado no próprio local em malha de $0,5 \mathrm{~mm}$ para a separação dos tanaidáceos. Os organismos assim obtidos foram acondicionados em baldes com água do local para o seu transporte até o Laboratório de Ecotoxicologia do CTTMar (UNIVALI).

Em laboratório, os organismos foram acondicionados em aquários de vidro contendo uma fina camada de sedimento e 6 litros de água do local de coleta, em densidades máximas de aproximadamente 400 indivíduos por aquário. Os lotes de organismos coletados foram divididos em dois grupos. Em um aquário os organismos foram aclimatados a salinidade de 15 (grupo 1), ajustando-se 3 unidades de salinidade a cada 3 horas, visto ser esta a salinidade utilizada para testes com substâncias de referências pela maioria dos autores. O grupo 2 foi mantido com a salinidade do local no momento de cada coleta (5,7-7,3-8,5-10-21) até a realização dos experimentos. Durante o período de aclimatação, que nunca ultrapassou dois dias, os aquários foram mantidos em temperatura controlada de $22 \pm 2{ }^{\circ} \mathrm{C}$, com fotoperíodo de $12 \mathrm{C}: 12 \mathrm{E}$ e aeração constante (Zamboni \& Costa, 2002).

\section{Testes com substâncias de referência}

Com uma pipeta de boca larga foram selecionados somente organismos saudáveis, entre 0,7 e $1,0 \mathrm{~cm}$, sendo excluídas as fêmeas ovígeras. Seguindo os procedimentos anteriores, para cada lote de organismos foram realizados experimentos em duas condições de salinidade, aclimatada a 15 no grupo 1 e igual à observada no momento da coleta no grupo $2(5,7-7,3-8,5-10-21)$.

Foram realizados um total de oito experimentos com o sulfato de zinco $\left(\mathrm{ZnSO}_{4} \cdot 7 \mathrm{H}_{2} \mathrm{O}\right)$, quatro no grupo 1 (salinidade 15) e quatro no grupo 2 (salinidade local). Os resultados obtidos no grupo 1 foram os utilizados para estabelecer a concentração letal e efetiva incipiente e o tempo mínimo de duração do experimento. A faixa de concentração testada nestes ensaios sempre esteve entre 640 e $5 \mathrm{mg} \mathrm{L}^{-1}$ de $\mathrm{Zn}$. Para o $\mathrm{CdCl}_{2}$ (4 a $0,031 \mathrm{mg} \mathrm{L}^{-1}$ de Cd), o DSS (dodecil sulfato de sódio) (320 a $5 \mathrm{mg} \mathrm{L}^{-1}$ ) e o $\mathrm{NH}_{4} \mathrm{Cl}\left(640\right.$ a $10 \mathrm{mg} \mathrm{L}^{-1}$ de $\mathrm{NH}_{4}$ ), foram executados um teste para cada condição de salinidade. A concentração de amônia não ionizada $\left(\mathrm{NH}_{3}\right)$ foi estimada por cálculo estequiométrico em base da temperatura, $\mathrm{pH}$ e salinidade da solução teste.

Os testes foram realizados de forma estática, com duração de 10 dias, sem alimentação, sem sedimento e sem aeração. Para cada concentração teste, incluindo o controle, foram preparadas 4 réplicas com 5 organismos cada e $125 \mathrm{~mL}$ de solução teste em salinidade adequada. Os experimentos foram conduzidos a $22 \pm 2{ }^{\circ} \mathrm{C}$ e fotoperíodo $12 \mathrm{C}: 12 \mathrm{E}$.

Os efeitos ou end points observados foram mortalidade e mobilidade. Para a mobilidade foi considerado efeito a incapacidade do organismo em reagir ao estímulo mecânico através da natação de qualquer velocidade ou enrolando seu corpo. Os experimentos foram acompanhados a cada 24 horas e os indivíduos mortos foram removidos. Dados de salinidade e pH foram anotados no início e no final dos experimentos. Os testes com substâncias de referência foram considerados aceitáveis quando a mortalidade ou a imobilidade total entre 
as réplicas do controle não foi superior a $20 \%$ (Zamboni \& Costa, 2002).

Os valores de $\mathrm{CE}_{50}$ (concentração efetiva para $50 \%$ dos organismos testados) e $\mathrm{CL}_{50}$ (concentração letal para $50 \%$ dos organismos testados) foram determinados através do método Trimmed Spearman-Karber utilizando-se o programa TSK versão 1.5 (EPA, Cincinnati, Ohio). As cartas-controle foram elaboradas segundo USEPA (2002). As comparações múltiplas entre os tempos de exposição foram realizadas mediante o uso da ANOVA para um nível de significância de $\mathrm{p}<0,05$ após a normalização dos dados segundo Zar (1996).

\section{RESULTADOS E DISCUSSÃO}

\section{Tempo de teste e end point}

O tempo mínimo de execução dos experimentos foi determinado a partir da estabilização dos valores de $\mathrm{CE}_{50}$ e $\mathrm{CL}_{50}$ para o $\mathrm{Zn}$ nos experimentos com organismos aclimatados $\mathrm{e}$ expostos a salinidade 15 (grupo 1). Os resultados demonstraram que foram necessários 6 dias para a estabilização dos valores de $\mathrm{CE}_{50}$ nos testes de mobilidade e 7 dias para a estabilização dos valores de $\mathrm{CL}_{50}$ para a mortalidade (Figura 1). Este tempo difere dos 4 dias que vêm sendo utilizados por outros autores e recomendados por Zamboni \& Costa (2002).

$\mathrm{O}$ valor da $\mathrm{CE}_{50}$ para o grupo 1 , após 6 dias de exposição ao zinco, foi de $5,39 \mathrm{mg} \mathrm{L}^{-1}$, enquanto que a $\mathrm{CL}_{50}$, mesmo com 7 dias de exposição, foi de $7,75 \mathrm{mg} \mathrm{L}^{-1}$ (Tabela 1). Segundo os resultados da ANOVA $(p<0,05)$ existe diferença significativa entre esses valores, e por isto, o modelo de experimento escolhido modificará o resultado final.

\section{Carta controle de sensibilidade}

As cartas de sensibilidade dos experimentos realizados demonstraram que todos os testes com zinco mantiveram-se dentro dos limites da amplitude de variação, porém os valores de $\mathrm{CE}_{50}(6$ dias) apresentaram maior variação em comparação com os de $\mathrm{CL}_{50}$ (7 dias) (Figure 2). Os coeficientes de variação (CV) obtidos para os testes de mortalidade $(<40 \%)$ e mobilidade $(<65 \%)$ (Tabela 1) confirmam a menor precisão para este último critério de efeito.
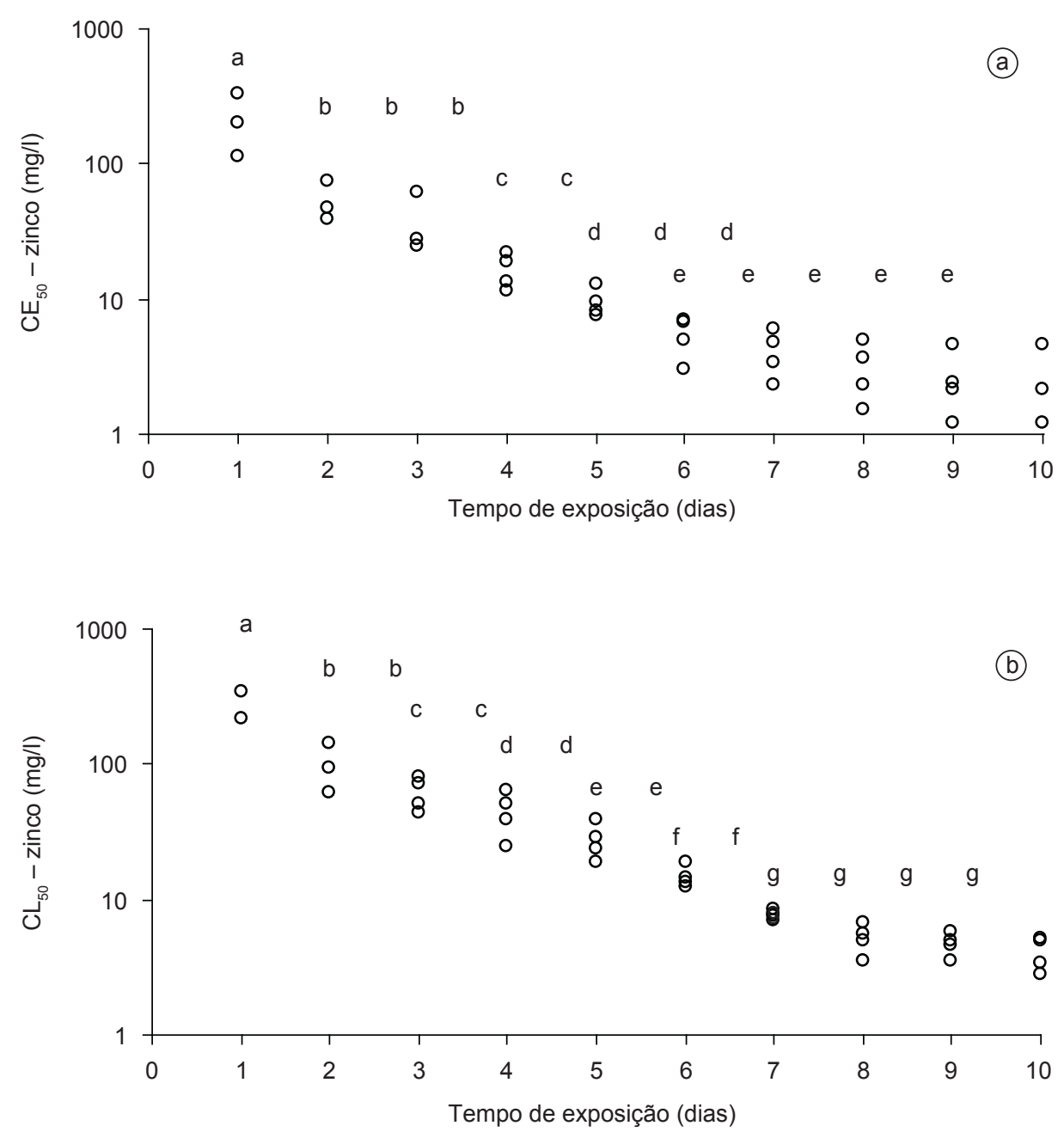

Figura 1 - Relação entre tempo de exposição e os valores de $\mathrm{CE}_{50}\left(\mathrm{~A}\right.$ - mobilidade) e $\mathrm{CL}_{50}\left(\mathrm{~B}\right.$ - mortalidade) sob efeito do zinco $\left(\mathrm{mg} . \mathrm{L}^{-1}\right)$ para os organismos aclimatados a salinidade de 15 . As letras indicam os tempos significativamente semelhantes segundo Teste de Tukey a posteriori para $\mathrm{p}<0,05$. 
Conforme a Figura 2, para o zinco, os valores de $\mathrm{CL}_{50}\left(7\right.$ dias) variaram entre $7,07 \mathrm{mg} \mathrm{L}^{-1}$ a $15,87 \mathrm{mg} \mathrm{L}^{-1}$ nas diferentes salinidades de exposição, sendo que os testes com salinidade 15 mostraram maior replicabilidade. Já os valores de $\mathrm{CE}_{50}$ (6 dias) ficaram entre $3 \mathrm{mg} \mathrm{L}^{-1}$ e 13,92 $\mathrm{mg} \mathrm{L}^{-1}$ de $\mathrm{Zn}$ para as mesmas salinidades.

\section{Sensibilidade do tanaidáceo Kalliapseudes schubartii em testes agudos}

A sensibilidade do tanaidáceo Kalliapseudes schubartii frente à amônia não ionizada $\left(\mathrm{NH}_{3}\right)$ e aos tóxicos de referência cádmio e o detergente dodecil sulfato de sódio (DSS) estão representados na Tabela 2. Para as três substâncias testadas, os valores de $\mathrm{CE}_{50}$ ( 6 dias) e $\mathrm{CL}_{50}$ ( 7 dias) mostraram-se semelhantes, ou seja, a mortalidade dos organismos não foi antecedida por uma evidente redução de mobilidade, diferente do que foi observado nos testes com o zinco.

Nos experimentos com o DSS o grupo que sofreu o processo de aclimatação para a salinidade de 15 apresentou maior sensibilidade. Para as demais substâncias, este ajuste de salinidade não se mostrou determinante para a sensibilidade dos organismos (Tabela 2). Os dois grupos apresentaram resultados semelhantes frente ao $\mathrm{NH}_{3}$, enquanto que para o cádmio o grupo na salinidade local $(8,5)$ mostrou maior sensibilidade. Para esta última substância, Costa (1997) também obteve maior sensibilidade de $K$. schubartii em experimentos de menor salinidade. Estes resultados ressaltam que o processo de aclimatação dos organismos pode interferir na sua sensibilidade, entretanto, sem um padrão aparente bem definido.
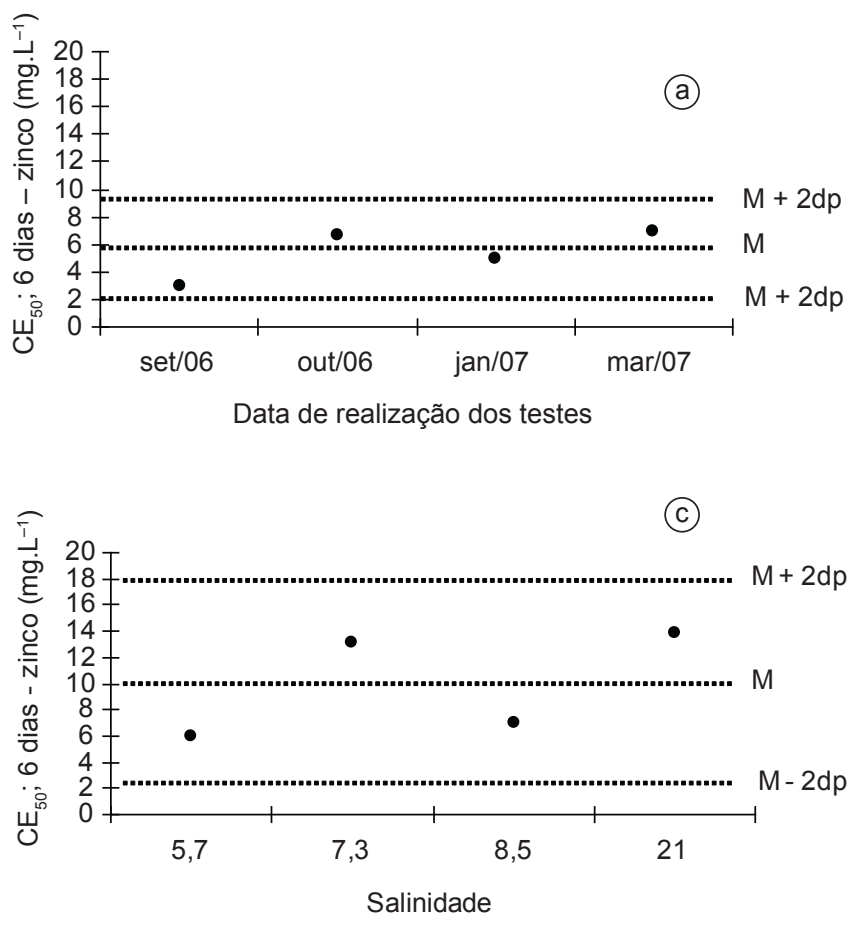

Tabela 1 - Valores médios de $\mathrm{CE}_{50}$ (mobilidade) e $\mathrm{CL}_{50}$ (mortalidade), desvio-padrão (DP) e coeficiente de variação $(\mathrm{CV})$ para o zinco $\left(\mathrm{mg} . \mathrm{L}^{-1}\right)$ utilizando organismos aclimatados e expostos a salinidade de $15 \mathrm{em}$ diferentes tempos de exposição.

\begin{tabular}{ccccccc}
\hline & \multicolumn{3}{c}{$\mathbf{C E}_{50}$} & & & $\mathbf{C L}_{50}$ \\
\hline Dia & Média & DP & CV & Média & DP & CV \\
\hline 1 & 213,91 & 106,11 & 49,60 & 283,35 & 92,79 & 32,75 \\
2 & 53,62 & 18,75 & 34,96 & 100,70 & 41,00 & 40,71 \\
3 & 38,10 & 21,06 & 55,27 & 61,95 & 16,98 & 27,41 \\
4 & 16,36 & 4,94 & 30,21 & 44,07 & 16,61 & 37,69 \\
5 & 9,53 & 2,40 & 25,13 & 27,74 & 9,01 & 32,50 \\
6 & 5,39 & 1,80 & 33,48 & 14,86 & 2,75 & 18,51 \\
7 & 4,14 & 1,63 & 39,27 & 7,75 & 0,55 & 7,09 \\
8 & 3,10 & 1,52 & 48,95 & 5,22 & 1,33 & 25,52 \\
9 & 2,58 & 1,41 & 54,62 & 4,71 & 0,90 & 19,16 \\
10 & 2,64 & 1,72 & 65,02 & 4,09 & 1,19 & 29,02 \\
\hline
\end{tabular}

Tabela 2 - Valores de $\mathrm{CE}_{50}$ (mobilidade) e $\mathrm{CL}_{50}$ (mortalidade) em mg.L $\mathrm{L}^{-1}$ para os ensaios realizados com o DSS, $\mathrm{NH}_{3}$ e Cd para os grupos 1 (salinidade de 15) e 2 (salinidades do local de coleta).

\begin{tabular}{lccc}
\hline Substância & Grupo & $\mathbf{C E}_{50}$ (6 dias) & $\mathbf{C L}_{50}$ (7 dias) \\
\hline DSS & $\mathbf{1}(15)$ & 12,75 & 12,12 \\
& $\mathbf{2}(5,7)$ & 25,42 & 26,39 \\
$\mathrm{NH}_{3}$ & $\mathbf{1}(15)$ & 3,31 & 3,16 \\
& $\mathbf{2}(10)$ & 3,60 & 3,77 \\
$\mathrm{Cd}$ & $\mathbf{1}(15)$ & 0,31 & 0,29 \\
& $\mathbf{2}(8,5)$ & 0,22 & 0,17 \\
\hline
\end{tabular}

*valores entre parênteses são as salinidades de aclimatação e teste.
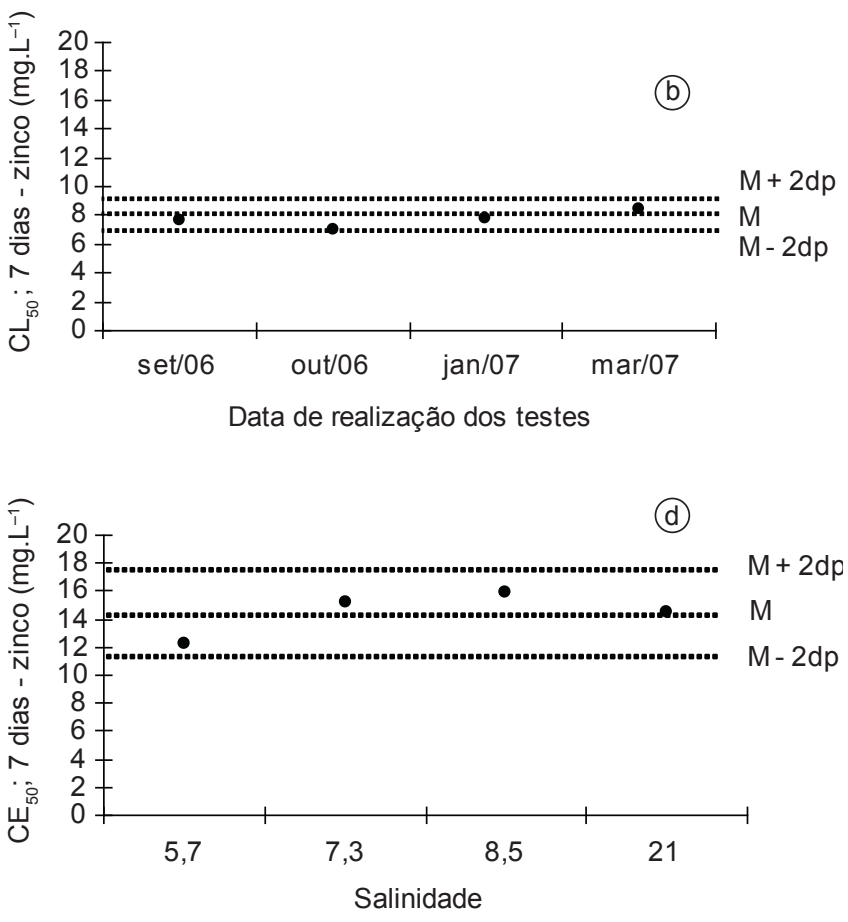

Figura 2 - Valores de $\mathrm{CE}_{50}$ e $\mathrm{CL}_{50}$ (ponto), média (M), limite superior $(\mathrm{M}+2 \mathrm{dp}$.) e inferior $(\mathrm{M}-2 \mathrm{dp}$.) para o grupo de organismos aclimatados e expostos a salinidade de 15 (A e B) e para o grupo aclimatado e testado com salinidade do local de coleta (C e D). 
Tabela 3 - Valores de $\mathrm{CL}_{50}$ (mortalidade) e $\mathrm{CE}_{50}$ (mobilidade) em mg.L $\mathrm{L}^{-1}$ para diferentes substâncias utilizando Kalliapseudes schubartii e três anfípodos utilizados em testes de toxicidade com sedimento segundo diferentes autores.

\begin{tabular}{|c|c|c|c|c|c|c|c|}
\hline Organismo-teste & Tempo de teste & Efeito & & DSS & Cd & $\mathrm{NH}_{3}$ & Autores \\
\hline Leptocheirus plumulosus (anfípodo) & $96 \mathrm{~h}$ & mortalidade & - & - & 1,06 & - & ASTM, 1992 \\
\hline Hyalella azteca (anfípodo) & $96 \mathrm{~h}$ & mortalidade & $0,073^{*}$ & - & $0,003-0,304$ & 0,126 & $\begin{array}{c}\text { Costa, } 1997 \\
\text { Besser et al., } 1998 \\
\text { USEPA, } 2000\end{array}$ \\
\hline Tiburonella viscana (anfípodo) & $48 \mathrm{~h}$ & mortalidade & 0,79 & 3,41 & - & - & Melo \& Nipper, 2007 \\
\hline \multirow[t]{4}{*}{$\begin{array}{l}\text { Kalliapseudes schubartii } \\
\text { (tanaidáceo) }\end{array}$} & $\begin{array}{l}96 \mathrm{~h} \\
96 \mathrm{~h}\end{array}$ & $\begin{array}{c}\text { mortalidade } \\
\text { mobilidade }\end{array}$ & $\begin{array}{c}- \\
16,36\end{array}$ & $\begin{array}{c}- \\
13,39\end{array}$ & $\begin{array}{c}0,105-0,937 \\
0,65\end{array}$ & $\begin{array}{c}- \\
4,35\end{array}$ & $\begin{array}{c}\text { Costa, } 1997 \\
\text { Presente trabalho }\end{array}$ \\
\hline & & mortalidade & 44,07 & 13,61 & 0,78 & 5,44 & \\
\hline & 6 dias & mobilidade & 5,39 & 12,75 & 0,31 & 3,31 & \\
\hline & 7 dias & mortalidade & 7,75 & 12,12 & 0,29 & 3,16 & \\
\hline
\end{tabular}

* teste de 10 dias de duração.

Na Tabela 3 estão representadas as sensibilidades de diferentes organismos bentônicos utilizados na avaliação da toxicidade de sedimentos estuarinos. De uma forma geral, o tanaidáceo K. schubartii apresenta uma maior resistência aos tóxicos de referência testados. Estas diferenças de sensibilidade reduzem quando é utilizado o tempo de teste de 7 dias estabelecido neste trabalho, mas ainda consistem como um fator limitante para sua utilização em testes de toxicidade.

Entretanto, a sua menor sensibilidade ao $\mathrm{NH}_{3}$ pode ser vantajosa na avaliação de sedimentos com altos teores de matéria orgânica, onde freqüentemente torna-se difícil reconhecer se a toxicidade sobre os organismos testados é causada pela presença de contaminantes ou por elevadas concentrações de nitrogênio amoniacal (Ho et al., 2002).

Outras espécies apresentadas na Tabela 3 apresentam algum ponto crítico em experimentos aplicados para ambientes estuarinos. Hyalella azteca, apesar de ser recomendado pela USEPA (2000), possui limitações para suportar salinidades elevadas. Segundo Melo \& Nipper (2007), Tiburonella viscana é uma espécie marinha que tolera salinidades próximas a 19, entretanto sua sobrevivência é afetada por sedimentos essencialmente finos, comuns em ambientes estuarinos.

A ampla faixa de tolerância do $K$. schubartii às variações de salinidade e a sua preferência por sedimentos finos, conferem a esta espécie, vantagens no seu uso em estudos e programas de monitoramento de ambientes estuarinos e lagunares.

\section{CONCLUSÃO}

O tempo mínimo para a execução dos testes de mortalidade, com o tóxico de referência Zn, utilizando Kalliapseudes schubartii foi de 7 dias, superior aos 4 dias que vêm sendo utilizados. Esta adequação do tempo de exposição a tóxicos de referências sugere a necessidade de estudos capazes de também estabelecer o tempo mínimo para experimentos com sedimento total, que atualmente são efetuados em 10 dias.

A utilização do efeito mobilidade não mostrou-se eficiente, visto que o tempo de execução dos experimentos diminuiu em apenas 1 dia e a variabilidade dos resultados foi superior aos testes de mortalidade.

Apesar de K. schubartii ser mais resistente que outras espécies empregadas nas avaliações de sedimentos, sua utilização ainda é necessária em locais que não apresentam uma melhor alternativa. Sua ampla tolerância às variações de salinidade, a preferência por sedimentos finos e maior resistência à amônia não ionizada são características que devem ser consideradas para sua escolha como organismo teste sobretudo em ambientes estuarinos.

\section{REFERÊNCIAS BIBLIOGRÁFICAS}

ABESSA, D.M.S., SOUZA, E.C.P.M., RACHID, B.R.F. \& MASTROTI, R.R., 1998, Use of the burrowing amphipod Tiburonella viscana as tool in marine sediments contamination assessment. Braz. Arch. Biol. Technol, 41(2): 225-230.

ABESSA, D.M.S., SOUZA, E.C.P.M., RACHID, B.R.F. \& MASTROTI, R.R., 2001, Toxicity of Sediments from Santos Estuary (SP, Brazil): Preliminary Results. Ecotox. Environ. Rest., 4(1): 6-9.

ASTM, 1992, Standard guide for conducting 10-day static sediment toxicity tests with marine and estuarine amphipods. Philadelphia. E 1367-92.

ASTM, 1995, Standard tests methods for measuring the toxicity of sediment-associated contaminants with freshwater invertebrates. Philadelphia. E 1706-95.

BESSER, J.M., INGERSOLL, C.G., LEONARD, E.N. \& MOUNT, D.R., 1998, Effect ofZeolite on Toxicity of Ammonia in Freshwater Sediments: Implications for Toxicity Identification Evaluation Procedures. Environ. Toxicol. Chem., 17(11): 2310-2317. 
CONSELHO NACIONAL DO MEIO AMBIENTE - CONAMA, 2004. Estabelece as diretrizes gerais e os procedimentos mínimos para a avaliação do material a ser dragado em águas jurisdicionais brasileiras. Resolução n. 344, de 25 de março de 2004. Brasília.

COSTA, J.B., 1997, Avaliação da adequabilidade de Kalliapseudes schubartii Mané-Garzón, 1922 (Crustacea: Tanaidacea) como organismo-teste para utilização em ensaios de toxicidade de sedimentos estuarinos. Dissertação (Bacharel em Oceanografia) FURG, 55p.

FIGUEIREDO, G.M. \& VIEIRA, J.P., 2005, Diel feeding, daily food consumption and the predatory impact of whitemouth croaker (Micropogonias furnieri) in an estuarine environment. Mar. Ecol., 26: 130-139.

FONSECA, D.B. \& D'INCAO, F., 2006, Mortality of Kalliapseudes schubartii in unvegetated soft bottoms of the estuarine region of the Lagoa dos Patos. Braz. Arch. Biol. Technol., 49: 257-261.

HO, K.T.; BURGESS, R.M.; PELLETIER, M.C.; SERBST, J.R.; RYBA, S.A.; CANTWELL, M.G.; KUHN,A.\& RACZELOWSKI, P., 2002, An overview of toxicant identification in sediments and dredged materials, Mar. Poll. Bull., 44: 286-293.

LEITE, F.P.P., 1995, Distribuiçãotemporale espacialde Kalliapseudes schubartii Mané-Garzón, 1949 (Crustacea,Tanaidacea) na região do Araçá, São Sebastião (SP). Arq. Biol. Tecnol., 38: 605-618.

LEITE, F.P.P., TURRA, A. \& SOUZA, E.C.F., 2003, Population biology and distribution of the tanaid Kalliapseudes schubartii Mané-Garzón, 1949, in an intertidal flat in southeastern Brazil. Braz. J. Biol., 63: 469-479.
MELO, S.L.R. \& NIPPER, M., 2007, Sediment toxicity tests using the burrowing amphipod Tiburonella viscana (Amphipoda: Platyischnopidae). Ecotox. Environ. Saf., 66: 412-420.

PAGLIOSA, P.R. \& BARBOSA, F.A.R., 2006, Assessing the environment-benthic fauna coupling in protected and urban areas of southern Brazil. Biol. Conserv., 129: 408-417.

PORTELA, C.M.S.; ARENZON, A. \& RAYA-RODRIGUEZ, M.T., 2006, Avaliação ecotoxicológica do sedimento do Arroio Sapucaia, RS, Brasil, através de ensaios de toxicidade utilizando diferentes amostradores e organismos-teste. J. Braz. Chem. Soc., 1: 119-122.

ROSA, L.C. \& BEMVENUTI, C.E., 2006. Temporal variability of the estuarine macrofauna of the Patos Lagoon, Brazil. Rev. Biol. Mar. Ocean., 41: 1-9.

USEPA. 2000, Methods for Measuring the Toxicity and Bioaccumulation of Sediment-associated Contaminants with Freshwater Invertebrates. 2 ed. Washington. EPA600-R-99-064.

USEPA. 2002, Short-term methods for estimating the chronic toxicity of effluents and receiving waters to marine and estuarine organisms. 3. ed. Washington. EPA-821-R-02-014.

ZAMBONI, A. J. \& COSTA, J.B., 2002, Testes de toxicidade com sedimentos marinhos utilizando tanaidáceos. In: NASCIMENTO, I.A.; SOUSA, E.C.P.M.; NIPPER, M. (eds.) Métodos em Ecotoxicologia Marinha: Aplicações no Brasil. Editora Artes Gráficas e Indústria Ltda, São Paulo, 262p.

ZAR, J.H., 1996, Biostatistical analysis. Prentice Hall, New Jersey, 3.ed., 662 p. 